\title{
Plasma Levels of Tumor Necrosis Factor Superfamily Molecules Are Increased in Bipolar Disorder
}

\author{
Izabela G. Barbosa ${ }^{1}$, Gabriela Neves Vaz ${ }^{1}$, Natalia Pessoa Rocha ${ }^{1,2}$, Rodrigo Machado-Vieira ${ }^{3}$, \\ Marcio Rogerio Diniz Ventura ${ }^{1}$, Rodrigo B. Huguet ${ }^{1}$, Moises E. Bauerr, Michael Berk ${ }^{5,6}$, Antônio L. Teixeira ${ }^{1,2}$ \\ ${ }^{1}$ Laboratório Interdisciplinar de Investigação Médica, Faculdade de Medicina, Universidade Federal de Minas Gerais (UFMG), Belo Horizonte, \\ Brazil, ${ }^{2}$ Neuropsychiatry Program, Department of Psychiatry and Behavioral Sciences, McGovern Medical School, The University of Texas Health \\ Science Center at Houston, Houston, TX, ${ }^{3}$ Experimental Therapeutics and Pathophysiology Branch, National Institute of Mental Health, Bethesda, \\ MD, USA, ${ }^{4}$ Laboratório de Imunologia do Envelhecimento, Instituto de Pesquisas Biomédicas, Pontifícia Universidade Católica do Rio Grande \\ do Sul, Porto Alegre, Brazil, ${ }^{5}$ Deakin University, IMPACT Strategic Research Centre, School of Medicine, Geelong, ${ }^{6}$ Orygen, The National Centre \\ of Excellence in Youth Mental Health, Department of Psychiatry and The Florey Institute of Neuroscience and Mental Health, The University \\ of Melbourne, Parkville, Australia
}

\begin{abstract}
Objective: Patients with bipolar disorder (BD) exhibit peripheral low-grade inflammation. The aim of the current study was to investigate the involvement of hitherto unexplored components of the tumor necrosis factor (TNF) superfamily in BD.

Methods: Eighty patients with type I BD and 50 healthy controls matched for age and gender were enrolled in this study. All subjects were assessed with the Mini-Plus to evaluate psychiatric comorbidities; the Young Mania Rating Scale and the Hamilton Depression Rating Scale to evaluate manic and depressive symptoms severity, respectively. TNF superfamily molecules (TNF, TNF-related weak inducer of apoptosis [TWEAK], TNF-related apoptosis-inducing ligand [TRAIL], soluble TNF receptor type 1 [sTNFR1], and soluble TNF receptor type 2 [sTNFR2]) levels were measured by ELISA.

Results: Patients with $\mathrm{BD}$, regardless of mood state, presented increased plasma levels of sTNFR1 and TWEAK in comparison with controls.
\end{abstract}

Conclusion: These findings corroborate the view that TNF superfamily may play a role in BD pathophysiology.

KEY WORDS: Bipolar disorder; TNF- $\alpha$; TWEAK; TRAIL; sTNFR.

\section{INTRODUCTION}

There is mounting evidence indicating that patients with bipolar disorder (BD) present peripheral low-grade inflammation. In this regard, one of the most consistently reported findings is tumor necrosis factor (TNF)- $\alpha$ activation represented by increased circulating levels of soluble TNF receptor type 1 (sTNFR 1$){ }^{1-4)}$ TNF- $\alpha$ dysregulation was also evidenced in the central nervous system (CNS) of patients with BD. A postmortem study found increased levels of transmembrane TNF- $\alpha$ in the anterior cingulate

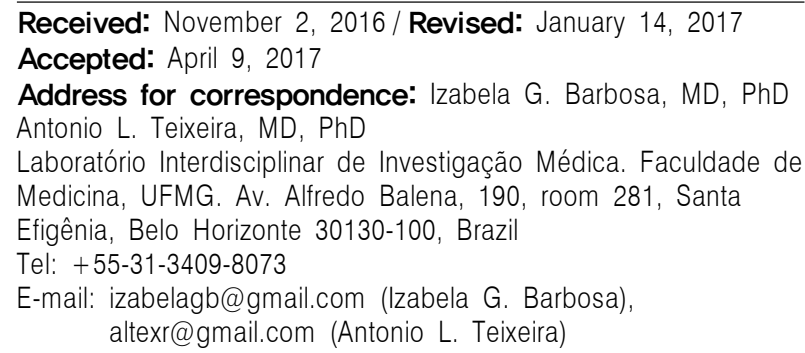

area and decreased levels of TNF- $\alpha$ receptor 2 mRNA in the dorsolateral prefrontal cortex, ${ }^{5)}$ regions implicated in executive functioning. ${ }^{6)}$ Interestingly, we have previously shown that impairment in executive functioning was associated with increased plasma levels of TNF- $\alpha$ and decreased plasma levels of soluble TNF receptor type 2 (sTNFR2) in patients with BD. ${ }^{7)}$

TNF- $\alpha$ is a cytokine that plays a key role in inflammatory responses. TNF- $\alpha$ acts by regulating the activity of immune cells and promoting the secretion of pro-inflammatory cytokines. The TNF superfamily includes several receptors and ligands (most of them in a soluble form) that regulate immune responses and apoptosis. Two specific receptors, TNFR1 (p55) and TNFR2 (p75), bind to TNF- $\alpha$ with high affinity, being responsible for translating into intracellular pathways TNF- $\alpha$ signaling. The TNFR1 and TNFR2 transmembrane receptors can be cleaved, and the extracellular domains can be released in the form of soluble receptors: sTNFR1 and sTNFR2, 
respectively. Blood levels of these two receptors can vary during inflammatory responses. As mentioned above, higher circulating levels of sTNFR1 were reported in patients with $\mathrm{BD}$, especially in mania, in comparison with controls. $^{1,8)}$

Other components of the TNF superfamily include the TNF-related weak inducer of apoptosis (TWEAK) and the TNF-related apoptosis-inducing ligand (TRAIL). TWEAK attenuates the transition from innate to adaptive Thelper 1 immune response, repressing pro-inflammatory cytokines like interferon- $\gamma$ and interleukin (IL)- 12 produced by natural killer cells and macrophages, and counterbalancing TNF- $\alpha$ activity. ${ }^{910)}$ TRAIL induces extrinsic apoptotic pathway by interacting with its receptors TRAIL-R1 and TRAIL-R2, also known as 'death receptors' ${ }^{11)}$ Accordingly, TRAIL is involved in immune homeostasis and tumor suppression. ${ }^{12)}$ TNF- $\alpha$, TRAIL, and TWEAK can also activate the NF-kappa B group of transcription factors which are implicated in the production of cytokines. ${ }^{13)}$

There is only one study that evaluated the involvement of TWEAK and TRAIL in psychiatric disorders. In this study, authors found decreased plasma levels of TWEAK in crack cocaine users in comparison with controls. In addition, TWEAK and TRAIL levels were associated with mood symptoms severity. ${ }^{12)}$ Recently, TWEAK was found capable to induce depressive symptoms in mice, being implicated on mood disorder pathophysiology. ${ }^{14)}$

To date, no study has been conducted in order to evaluate the levels of the TNF superfamily molecules TWEAK and TRAIL in patients with BD. Therefore, the aim of the current study was to investigate the involvement of hitherto unexplored components of the TNF superfamily in BD. We hypothesize that the patients with BD present increased plasma levels of TNF superfamily components in comparison with controls.

\section{METHODS}

Eighty patients with type $1 \mathrm{BD}$ and 50 aged-matched controls were enrolled in this study. Patients were consecutively recruited from the Mood Disorders Outpatient Clinic at Governador Israel Pinheiro Hospital, Belo Horizonte, Brazil. BD diagnosis was confirmed independently by two psychiatrists according to the Mini-International Neuropsychiatric Interview (MINI-Plus). ${ }^{15)}$ Psychiatric comorbidities were also evaluated by the MINI-Plus ${ }^{15)}$ interview and were not considered as exclusion criteria. All patients were assessed by the Young Mania Rating Scale (YMRS) ${ }^{16)}$ and the Hamilton Depression Rating Scale
(HDRS $)^{17)}$ in order to evaluate the severity of manic and depressive symptoms, respectively. Remission was defi ned by YMRS score $<7$ and HDRS score $<7$ points for at least eight consecutive weeks.

Control group was recruited from the local population and it was composed of subjects without any psychiatric comorbidity, family history of psychiatry disease, suicide behavior or cognitive deficit. Controls were subjected to MINI-Plus interview to exclude psychiatric disorders.

Subjects with dementia, infectious or autoimmune diseases, or who had used steroids, anti-inflammatory drugs, or antibiotics within four weeks previous to the evaluation were excluded from this research protocol. The study was approved by the local ethic committees (COEP of Universidade Federal de Minas Gerais; CAAE.26363614.0. 0000.5149). All participants signed written informed consent prior to study participation.

Ten milliliters of blood were drawn by venipuncture into a vacuum tube containing heparin at the same day of the clinical assessment (between 8 to 10 a.m.). Blood was immediately centrifuged twice at $1,800 \mathrm{~g}$ for 10 minutes. Plasma was collected and stored at $-70^{\circ} \mathrm{C}$ until assayed. Plasma levels of TNF- $\alpha$ (catalog number: DY210), sTNFR1 (catalog number: DY225), sTNFR2 (catalog number: DY726), TWEAK (catalog number: DY1090), and TRAIL (catalog number: DY375) were measured by enzymelinked immunosorbent assay (ELISA) according to the procedures supplied by the manufacturer (DuoSet; R\&D Systems, Minneapolis, MN, USA). All molecules were measured in duplicate and experiments were performed blinded regarding the participants' diagnosis. Concentrations are expressed as $\mathrm{pg} / \mathrm{ml}$.

Descriptive statistics were used to report socio-demographic and clinical features of the sample. All variables were tested for normal distribution by means of the Kolmogorov-Smirnov test. Differences between two groups (patients vs. controls or BD in remission vs. BD in mania) were assessed by Mann-Whitney or Student $t$ tests when non-normally or normally distributed, respectively. Spearman's correlation analyses were performed to evaluate the association between TWEAK, TRAIL, TNF- $\alpha$, sTNFR1, and sTNFR2 levels and age, length of illness, YMRS, HDRS scores. All statistical tests were two tailed and used a significance level of $p<0.05$. Statistical analyses were performed using SPSS software version 17.0 (SPSS Inc., Chicago, IL, USA). 
Table 1. Demographic and clinical features of controls and bipolar disorder (BD) patients

\begin{tabular}{lccc}
\hline \multicolumn{1}{c}{ Feature } & Control $(n=50)$ & BD patient $(n=80)$ & $p$ value \\
\hline Age $(y r)$ & $46.80 \pm 9.47$ & $46.09 \pm 12.32$ & $0.38^{*}$ \\
Sex, male/female & $15 / 35$ & $52 / 80$ & 0.58 \\
Length of illness $(y r)$ & - & $22.93 \pm 12.66$ & - \\
YMRS score & - & $13.78 \pm 13.44$ & - \\
HDRS score & - & $3.70 \pm 4.65$ & - \\
Medications in use & & & \\
Lithium & - & $43(53.8)$ & - \\
Antipsychotics & - & $49(61.3)$ & - \\
Anticonvulsants & - & $39(48.8)$ & - \\
\hline
\end{tabular}

Values are presented as mean \pm standard deviation, number only, or number (\%).

YMRS, Young Mania Rating Scale; HDRS, Hamilton Depression Rating Scale.

*Mann Whitney test.

\section{RESULTS}

Demographic and clinical features of the study populations are shown in Table 1. Control subjects and patients with $\mathrm{BD}$ presented comparable age and gender. Among 80 patients with BD, 38 were in remission (11 male, 27 female; mean \pm standard deviation [SD] of age $46.37 \pm 11.15$ years) and 42 in mania ( 17 male, 25 female; age $51.07 \pm 13.02$ years). The mean $( \pm \mathrm{SD})$ length of illness was $22.68( \pm 11.08)$ years in patients in remission and $23.19( \pm 14.26)$ years in patients in mania. There were no significant differences between patients in remission and mania regarding age, gender and disease length. Patients with BD in mania presented YMRS and HDRS of 25.36 $( \pm 7.48)$ and $5.09( \pm 5.49)$, respectively. Patients with BD in remission presented YMRS and HDRS of $1.00( \pm 1.97)$ and $2.16( \pm 2.82)$, respectively. Forty-five out of 80 patients with BD $(56.3 \%)$ were in use of at least two different mood stabilizer drugs. The information about mood stabilizers in use is shown in Table 1.

We observed changes in plasma levels of TNF family molecules in BD. Patients with BD exhibited increased plasma levels of sTNFR1 (Fig. 1A) and TWEAK (Fig. 1C) in comparison with controls. We did not find differences between patients with $\mathrm{BD}$ and controls regarding plasma levels of sTNFR2, TRAIL and TNF (Fig. 1A, 1D, and $1 \mathrm{E}$, respectively). We found no significant differences when comparing the levels of TNF family markers between patients with BD in remission and in mania (Fig. 2A-2F).

In patients with $\mathrm{BD}$, TWEAK plasma levels positively correlated with TNF- $\alpha$ plasma levels $(\rho=0.528, p<$ $0.001)$ and with TRAIL plasma levels $(\rho=0.621, p<$ $0.001)$. TNF- $\alpha$, TRAIL, and TWEAK plasma levels did not correlate with age, length of disease, YMRS and HDRS scores. TWEAK and TRAIL plasma levels were not associated with the presence of psychiatric and clinical co-morbidities, nicotine dependence, or the use of any mood-stabilizing drug (i.e., atypical antipsychotics, lithium or anticonvulsants). When considering controls, there was a positive correlation between TWEAK and Trail plasma levels $(\rho=0.297, p=0.04)$.

\section{DISCUSSION}

This is the first study to evaluate the TNF superfamily molecules TWEAK and TRAIL in BD. Patients with BD presented increased plasma levels of TWEAK and sTNFR1 in comparison with controls. The changes in TWEAK and sTNFR1 levels might be a trait marker of $\mathrm{BD}$, since patients in different mood states (i.e., patients in mania and patients in remission) did not present any difference in the levels of the evaluated markers. The current results corroborate the view of chronic low-grade inflammation in $\mathrm{BD}$, indicating the involvement of the TNF superfamily.

TWEAK plasma levels were increased in BD patients regardless their mood state. ${ }^{18)}$ TWEAK has the potential to promote tissue remodeling through its ability to orchestrate inflammatory, angiogenic and/or fibrogenic processes, and regulate parenchymal cell survival and growth. ${ }^{8,10)}$ During acute mood episodes, like mania, in parallel with increase in pro-inflammatory stimuli, ${ }^{8,19)}$ TWEAK levels may increase in order to control or even counterbalance this process. During remission, low grade inflammation might also lead to persistent TWEAK pathway activation. Theoretically, even this mild chronic inflammation may drive and/or be associated with progressive CNS damage and pathological tissue remodeling. ${ }^{20,21)}$ It remains to be determined whether TWEAK increase is specific for patients with BD or this phenomenon is observed in other major psychiatric diseases.

We did not find any significant difference between patients with BD and controls regarding plasma levels of TRAIL. Similar to our study, a previous study involving patients with crack cocaine abstinence and controls did not report difference in TRAIL plasma levels between groups. ${ }^{12)}$ TRAIL is not expressed under physiological conditions in the CNS, and its role in regulating inflammation and apoptosis in the CNS needs to be further explored. $^{22)}$

TNF- $\alpha$ activation is consistently reported in BD. For 

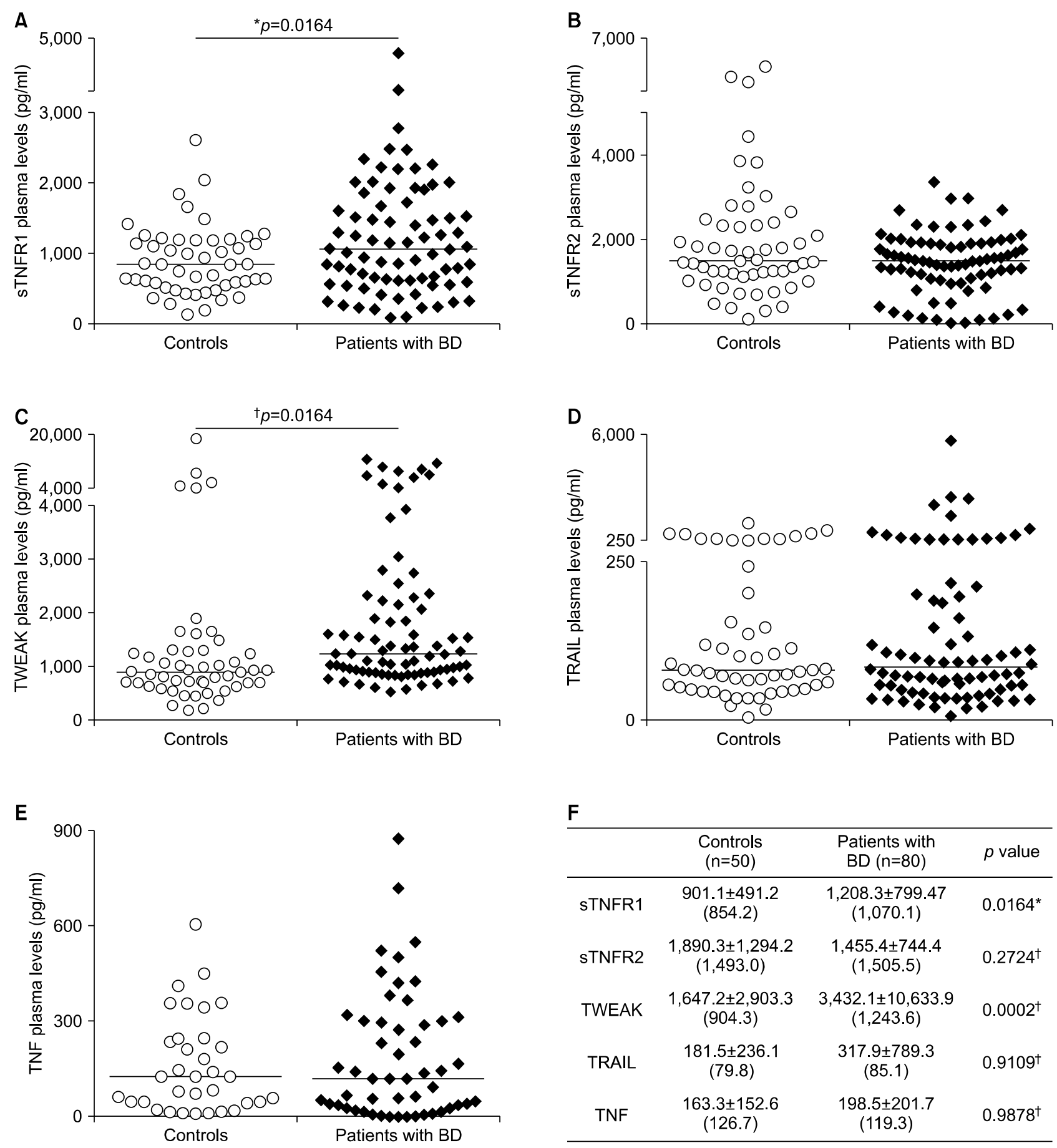

\begin{tabular}{|c|c|c|c|}
\hline & $\begin{array}{l}\text { Controls } \\
(n=50)\end{array}$ & $\begin{array}{l}\text { Patients with } \\
\text { BD }(n=80)\end{array}$ & $p$ value \\
\hline sTNFR1 & $\begin{array}{c}901.1 \pm 491.2 \\
(854.2)\end{array}$ & $\begin{array}{c}1,208.3 \pm 799.47 \\
(1,070.1)\end{array}$ & $0.0164^{*}$ \\
\hline sTNFR2 & $\begin{array}{c}1,890.3 \pm 1,294.2 \\
(1,493.0)\end{array}$ & $\begin{array}{c}1,455.4 \pm 744.4 \\
(1,505.5)\end{array}$ & $0.2724^{\dagger}$ \\
\hline TWEAK & $\begin{array}{c}1,647.2 \pm 2,903.3 \\
(904.3)\end{array}$ & $\begin{array}{c}3,432.1 \pm 10,633.9 \\
(1,243.6)\end{array}$ & $0.0002^{\dagger}$ \\
\hline TRAIL & $\begin{array}{l}181.5 \pm 236.1 \\
\quad(79.8)\end{array}$ & $\begin{array}{c}317.9 \pm 789.3 \\
(85.1)\end{array}$ & $0.9109^{\dagger}$ \\
\hline TNF & $\begin{array}{c}163.3 \pm 152.6 \\
(126.7)\end{array}$ & $\begin{array}{c}198.5 \pm 201.7 \\
\quad(119.3)\end{array}$ & $0.9878^{\dagger}$ \\
\hline
\end{tabular}

Fig. 1. Plasma levels of TNF superfamily molecules in patients with bipolar disorder (BD) and controls. Patients with BD presented higher levels of sTNFR1 (A) and TWEAK (C) than controls. No differences between groups were observed regarding plasma levels of sTNFR2 (B), TRAIL (D), and TNF (E). Plasma levels ( $\mathrm{pg} / \mathrm{ml}$ ) of all measured molecules (mean \pm standard deviation (median)) and statistics are provided in (F). TNF, tumor necrosis factor; STNFR1, soluble TNF receptor type 1; sTNFR2, soluble TNF receptor type 2; TWEAK, TNF-related weak inducer of apoptosis; TRAIL, TNF-related apoptosis-inducing ligand.

${ }^{*}$ Student's $t$-test, ${ }^{\dagger}$ Mann-Whitney test. Horizontal bars represent the medians.

instance, Fiedorowicz et al. ${ }^{23)}$ showed increased circulating levels of TNF- $\alpha$, as well as its soluble receptorssTNFR1/sTNFR2-in mania. Similarly, Pandey et al. ${ }^{24)}$ described high mRNA levels of TNF- $\alpha$, TNFR1 and IL-1R1 in BD. TNF- $\alpha$ degrades soon after release, partly explaining the fact that this molecule is not necessarily 

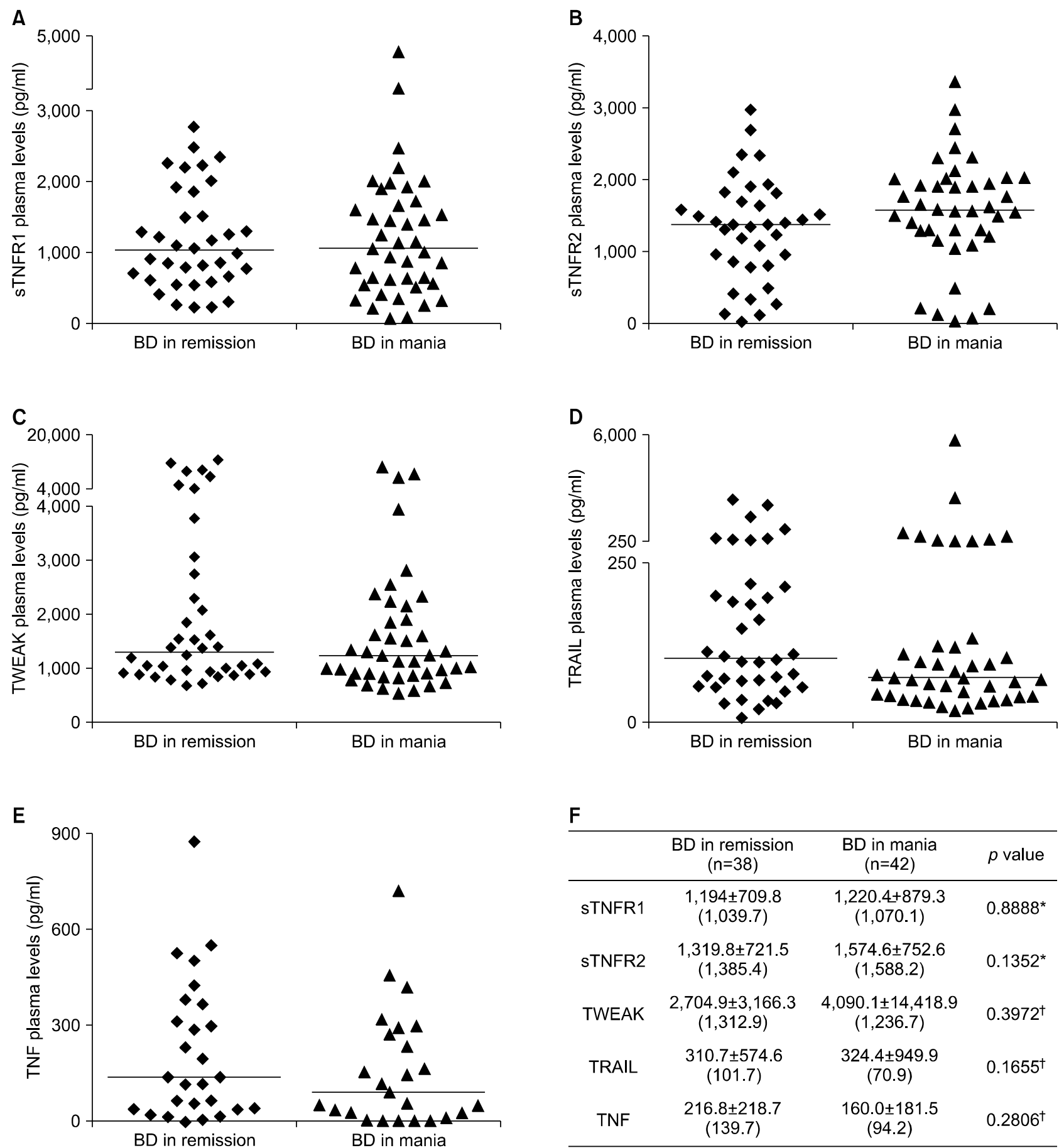

\begin{tabular}{|c|c|c|c|}
\hline & $\begin{array}{c}\text { BD in remission } \\
(n=38)\end{array}$ & $\begin{array}{c}\mathrm{BD} \text { in mania } \\
(\mathrm{n}=42)\end{array}$ & $p$ value \\
\hline sTNFR1 & $\begin{array}{c}1,194 \pm 709.8 \\
(1,039.7)\end{array}$ & $\begin{array}{c}1,220.4+879.3 \\
(1,070.1)\end{array}$ & $0.8888^{*}$ \\
\hline sTNFR2 & $\begin{array}{c}1,319.8 \pm 721.5 \\
(1,385.4)\end{array}$ & $\begin{array}{c}1,574.6 \pm 752.6 \\
(1,588.2)\end{array}$ & $0.1352^{*}$ \\
\hline TWEAK & $\begin{array}{c}2,704.9 \pm 3,166.3 \\
(1,312.9)\end{array}$ & $\begin{array}{c}4,090.1 \pm 14,418.9 \\
(1,236.7)\end{array}$ & $0.3972^{\dagger}$ \\
\hline TRAIL & $\begin{array}{c}310.7 \pm 574.6 \\
(101.7)\end{array}$ & $\begin{array}{c}324.4 \pm 949.9 \\
\quad(70.9)\end{array}$ & $0.1655^{\dagger}$ \\
\hline TNF & $\begin{array}{c}216.8 \pm 218.7 \\
(139.7)\end{array}$ & $\begin{array}{c}160.0 \pm 181.5 \\
(94.2)\end{array}$ & $0.2806^{\dagger}$ \\
\hline
\end{tabular}

Fig. 2. Plasma levels of TNF superfamily molecules in patients with bipolar disorder $(B D)$ in remission and in mania. Patients with $B D$ in remission and in mania presented similar plasma levels of sTNFR1 (A), sTNFR2 (B), TWEAK (C), TRAIL (D), and TNF (E). Plasma levels (pg/ml) of all measured molecules (mean \pm standard deviation (median)) and statistics are provided in (F).

TNF, tumor necrosis factor; STNFR1, soluble TNF receptor type 1; sTNFR2, soluble TNF receptor type 2; TWEAK, TNF-related weak inducer of apoptosis; TRAlL, TNF-related apoptosis-inducing ligand.

${ }^{*}$ Student's -test, ${ }^{\dagger}$ Mann-Whitney test. Horizontal bars represent the medians.

found altered in BD case series. ${ }^{8)}$ It is worth mentioning that inconsistencies in the literature might also arise from the heterogeneity of BD samples in terms of clinical fea- tures and immune profile. Anyway, TNF- $\alpha$ levels positively correlated with TWEAK and our results reinforce the hypothesis that the TNF system is activated in BD. 
This study has strengths and limitations that must be considered when interpreting the results. The diagnostic interviews of patients and controls were performed using the same protocol. In addition, the exclusion of patients with medical conditions such as inflammatory diseases can be regarded as strength of the study. The lack of strict control for confounding factors, such as body mass index, medications in use, and tobacco use must be considered limitations, as well as the cross-sectional nature of the study. In-vitro studies have demonstrated that mood stabilizers, antipsychotics, and antiepileptic drugs influence the production of inflammatory markers by immune cells. $^{25,26)}$ Our results, however, were not influenced by medication in use. In addition, it would have been informative to evaluate the association between functionality and the TNF molecules' levels and to have a bipolar depression only arm as well. The fact that other molecules from the TNF superfamily were not investigated is also a limitation of our study.

In conclusion, our findings reinforce the view that inflammatory dysfunction is present in BD and that the TNF superfamily, particularly TWEAK, may play a role in the pathophysiology of BD. Given the role of TWEAK as an apoptosis regulator, this study has implications for the understanding the pathways to progressive CNS structural changes in BD.

\section{- Acknowledgments}

Authors would like to acknowledge the participation of volunteers in this study. We also appreciate the critical comments and expert technical assistance of members of our collaborative research groups. This work was funded by the Brazilian government agencies CAPES, CNPq and Fapemig. The funding sources had no influence on study design, data analysis, interpretation of results, writing of the manuscript, or the decision to submit the paper for publication.

\section{REFERENCES}

1. Modabbernia A, Taslimi S, Brietzke E, Ashrafi M. Cytokine alterations in bipolar disorder: a meta-analysis of 30 studies. Biol Psychiatry 2013;74:15-25.

2. Munkholm K, Braüner JV, Kessing LV, Vinberg M. Cytokines in bipolar disorder vs. healthy control subjects: a systematic review and meta-analysis. J Psychiatr Res 2013;47:1119-1133.

3. Munkholm K, Vinberg M, Vedel Kessing L. Cytokines in bipolar disorder: a systematic review and meta-analysis. $J$ Affect Disord 2013;144:16-27.

4. Teixeira AL, de Sousa RT, Zanetti MV, Brunoni AR, Busatto GF, Zarate CA Jr, et al. Increased plasma levels of soluble TNF receptors 1 and 2 in bipolar depression and impact of lithium treatment. Hum Psychopharmacol 2015;30:52-56.

5. Dean B, Gibbons AS, Tawadros N, Brooks L, Everall IP, Scarr E. Different changes in cortical tumor necrosis factor$\alpha$-related pathways in schizophrenia and mood disorders. Mol Psychiatry 2013;18:767-773.

6. Townsend JD, Torrisi SJ, Lieberman MD, Sugar CA, Bookheimer SY, Altshuler LL. Frontal-amygdala connectivity alterations during emotion downregulation in bipolar I disorder. Biol Psychiatry 2013;73:127-135.

7. Barbosa IG, Rocha NP, Huguet RB, Ferreira RA, Salgado $\mathrm{JV}$, Carvalho LA, et al. Executive dysfunction in euthymic bipolar disorder patients and its association with plasma biomarkers. J Affect Disord 2012;137:151-155.

8. Barbosa IG, Huguet RB, Mendonça VA, Sousa LP, Neves FS, Bauer ME, et al. Increased plasma levels of soluble TNF receptor I in patients with bipolar disorder. Eur Arch Psychiatry Clin Neurosci 2011;261:139-143.

9. Maecker H, Varfolomeev E, Kischkel F, Lawrence D, LeBlanc $\mathrm{H}$, Lee $\mathrm{W}$, et al. TWEAK attenuates the transition from innate to adaptive immunity. Cell 2005;123:931-944.

10. Croft M. The TNF family in $T$ cell differentiation and function--unanswered questions and future directions. Semin Immunol 2014;26:183-190.

11. Cullen SP, Martin SJ. Fas and TRAIL 'death receptors' as initiators of inflammation: Implications for cancer. Semin Cell Dev Biol 2015;39:26-34.

12. Levandowski ML, Viola TW, Wearick-Silva LE, Wieck A, Tractenberg SG, Brietzke E, et al. Early life stress and tumor necrosis factor superfamily in crack cocaine withdrawal. $J$ Psychiatr Res 2014;53:180-186.

13. Mas S, Martínez-Pinna R, Martín-Ventura JL, Pérez R, Gomez-Garre D, Ortiz A, et al. Local non-esterified fatty acids correlate with inflammation in atheroma plaques of patients with type 2 diabetes. Diabetes 2010;59:1292-1301.

14. Wen J, Chen CH, Stock A, Doerner J, Gulinello M, Putterman C. Intracerebroventricular administration of TNF-like weak inducer of apoptosis induces depression-like behavior and cognitive dysfunction in non-autoimmune mice. Brain Behav Immun 2016;54:27-37.

15. Sheehan DV, Lecrubier Y, Sheehan KH, Amorim P, Janavs J, Weiller E, et al. The Mini-International Neuropsychiatric Interview (M.I.N.I.): the development and validation of a structured diagnostic psychiatric interview for DSM-IV and ICD-10. J Clin Psychiatry 1998;59 Suppl 20:22-33;quiz 34-57.

16. Young RC, Biggs JT, Ziegler VE, Meyer DA. A rating scale for mania: reliability, validity and sensitivity. Br J Psychiatry 1978;133:429-435.

17. Hamilton M. Development of a rating scale for primary depressive illness. Br J Soc Clin Psychol 1967;6:278-296.

18. Davis J, Maes M, Andreazza A, McGrath JJ, Tye SJ, Berk M. Towards a classification of biomarkers of neuropsychiatric disease: from encompass to compass. Mol Psychiatry 2015;20:152-153.

19. Kapczinski F, Dal-Pizzol F, Teixeira AL, Magalhaes PV, Kauer-Sant'Anna M, Klamt F, et al. Peripheral biomarkers and illness activity in bipolar disorder. $J$ Psychiatr Res 2011;45:156-161.

20. Berk M, Berk L, Dodd S, Cotton S, Macneil C, Daglas R, et al. Stage managing bipolar disorder. Bipolar Disord 2014;16:471-477.

21. Barbosa IG, Bauer ME, Machado-Vieira R, Teixeira AL. Cytokines in bipolar disorder: paving the way for neuroprogression. Neural Plast 2014;2014:360481. 
22. Griffiths MR, Gasque P, Neal JW. The multiple roles of the innate immune system in the regulation of apoptosis and inflammation in the brain. J Neuropathol Exp Neurol 2009; 68:217-226

23. Fiedorowicz JG, Prossin AR, Johnson CP, Christensen GE, Magnotta VA, Wemmie JA. Peripheral inflammation during abnormal mood states in bipolar I disorder. J Affect Disord 2015;187:172-178.

24. Pandey GN, Ren X, Rizavi HS, Zhang H. Abnormal gene expression of proinflammatory cytokines and their receptors in the lymphocytes of patients with bipolar disorder. Bipolar Disord 2015;17:636-644.

25. Himmerich H, Schönherr J, Fulda S, Sheldrick AJ, Bauer $\mathrm{K}$, Sack U. Impact of antipsychotics on cytokine production in-vitro. J Psychiatr Res 2011;45:1358-1365.

26. Himmerich H, Bartsch S, Hamer H, Mergl R, Schönherr J, Petersein $\mathrm{C}$, et al. Impact of mood stabilizers and antiepileptic drugs on cytokine production in-vitro. J Psychiatr Res 2013;47:1751-1759. 